\title{
Lynn Hunt, Inventing Human Rights, A History
}

New York et Londres, W.W. Norton et Cie, 2007, 272 p., ISBN

0-393-06095-0, 25,95 \$.

Jean-Clément Martin

\section{Q OpenEdition}

1 Journals

Édition électronique

URL : https://journals.openedition.org/ahrf/11412

DOI : $10.4000 /$ ahrf.11412

ISSN : 1952-403X

Éditeur :

Armand Colin, Société des études robespierristes

Édition imprimée

Date de publication : 1 mars 2008

Pagination : 216-218

ISSN : 0003-4436

Référence électronique

Jean-Clément Martin, «Lynn Hunt, Inventing Human Rights, A History », Annales historiques de la

Révolution française [En ligne], 351 | janvier-mars 2008, mis en ligne le 29 décembre 2009, consulté le 03 août 2021. URL : http://journals.openedition.org/ahrf/11412; DOI : https://doi.org/10.4000/ahrf. 11412 
système, se résigne. On a même pu faire admettre au Puy-du-Fou une partie des thèses réactionnaires en s'appuyant sur la recherche de racines effectivement détruites par la concentration capitaliste.

Pourtant, par là subsiste un autre aspect de la Révolution, des refus lors des référendums européens de 1992 et 2005, sont réapparus, selon une géographic qui rappelle les clivages anciens. Plus large que l'idéc de libération, la notion d'émancipation connaît un certain succès et avec elle reparaît l'idée, venue des Lumières et de la Révolution, de progrès, mais non pas comme un phénomène linéaire et continu, comme au contraire un mouvement doublement contradictoire : fait d'une alternance d'avancées et de reculs et, dans son contenu composé en même temps d'aspects positifs et de régressions.

Ces quelques notations à la lecture du livre suggèrent que la Révolution n'est en fait toujours pas un objet froid et vit encore, et l'un des acteurs de cette survivance est bien l'auteur, à la fois par son ouvre scientifique et par les charges qu'il a assurées à la tête de l'Institut d'Histoire de la Révolution Française de la Sorbonne, à la présidence de la Société des études robespierristes et à celle de la Commission CNRS ad hoc, en particulier dans la presse et les embúches du bicentenaire. Comme cet ouvrage en porte une preuve, chacun se souvient que ces fonctions ne consistèrent pas seulement à inaugurer les chrysanthèmes.

Guy LEMARCHAND

Lynn Hunt, Inventing Human Rights, A History, New York et Londres, W.W. Norton et Cie, 2007, 272 p., ISBN 0-393-06095-0, 25,95\$.

Lynn Hunt propose ici un ouvrage original pour parler des droits de l'homme en partant de l'expérience historique du XVIII' siècle en Europe et en Amérique du Nord et en allant jusqu'à l'usage de ces droits au $\mathrm{XX}^{\mathrm{c}}$ siècle. Elle montre les étapes par lesquelles la conscience collective s'est cmparée de cette notion jusqu'à en faire un des points essentiels de la culture occidentale. Il ne s'agit pas d'une histoire téléologique ou hagiographique, mais de la reconstitution de la démarche empirique commune à différents États, la France, la Grande-Bretagne, les États-Unis, au travers des modifications des sensibilités, pour donner à voir les conditions de l'application historique d'une notion.

Lintroduction est significative en comparant les deux versions du préambule de la Déclaration d'indépendance des États-Unis de 1776 qui passent d'une formulation marquée par le sentimentalisme de l'époque à une expression pleinement politique, instituée dans l'univers politique. Cette prise de conscience est le tournant qui fait passer la définition des droits du flou dans lequel ils se trouvent encore à la moitić du XVIII" siècle à une clarté inexorable. Cette imposition de l'évidence des droits eux-mêmes s'est réalisée par une mutation des sensibilités, via notamment la littérature romanesque. Le résultat aura été de rendre la torture judiciaire insupportable à l'opinion. Dans une approche pragmatique et pratique, Lynn Hunt insiste sur le fait que ce changement n'est pas lié à une quelconque imprégnation informelle des idées, mais qu'il s'agit bien d'une modification de la collectivité des lecteurs, convaincus par les émotions ressenties et partagées qu'ils peuvent doréna- 
vant intervenir eux-mêmes sur le monde. La sécularisation du gouvernement et la descente sur terre des mystères du pouvoir n'a pas été une opération miraculeuse ou provoquéc par les philosophes ; c'est, au contraire, la modification du groupe social lui-même qui s'est accompagnée, logiquement, d'une mutation dans les représentations et donc d'exigences inédites. La diffusion des romans épistolaires permet ainsi de comprendre comment s'est imposée peu à peu la conviction de pouvoir être soi-méme acteur de sa vie et contester le principe d'autorité. Les personnages féminins sont de ce point de vue exemplaires dans cette évolution, que ce soit Julie, Clarissa et Paméla, autant par l'écho que ces héroïnes possèdent que par la fermeture sociale qui est mise en place contre toute tentative féminine d'émancipation. Cependant c'est bien ce passage du corps sacré religieux au corps sacré en lui-même, destiné à être contrôlé, qui créc les conditions par lesquelles les exécutions publiques deviennent insupportables. Une nouvelle conception de l'humanité touche ainsi les élites cultivées de l'Europe, ce qu'illustrent les affaires judiciaires célèbres qui scandent cette montée du refus de l'État violent.

La mise en place pratique des droits de l'homme résultant de la rencontre factuelle de la guerre d'indépendance américaine comme des conflits politiques français, permet de comprendre que, ces conditions n'existant pas sur le sol de la Grande-Bretagne, celle-ci n'est pas impliquée par cette mutation, qui l'a touchéc seulement dans les sensibilités et les débats intellectuels. Ce sont les trames événementielles qui créent les conditions par lesquelles les trois pays connaissent des évolutions séparées et même opposées, alors que jusque là ils avaient participé au même climat. Les déclarations aux États-Unis n'obtiennent pas pour cette raison l'importance que la Déclaration de 1789 aura à partir de la France. Pour des raisons factuelles, les tendances universalistes ne peuvent pas trouver d'expression complète hors de France si bien que le récit de Lynn Hunt se consacre alors à l'histoire française, où la « déclaration » des droits se pose comme une prise de pouvoir, même si la langue des «droits » telle qu'elle est dorénavant parlée en France devient un modèle européen. Une des conséquences notables est l'abolition de tortures judiciaires et de toute atteinte à l'honneur des citoyens, qui traduit concrètement un changement important des mentalités. Si l'honncur féminin demeure dans le domaine du privé, l'honneur des hommes devient commun à tous, roturiers ou nobles, et est lié à leurs actions publiques. Dans la suite logique de cette innovation et de sa limite, les droits s'appliquent aux différents groupes confessionnels et ethniques - avec plus ou moins de rapidité - mais ne sont pas étendus aux femmes, dont les prétentions, pourtant fondées sur les mutations des sensibilités, étaient évidentes. Mais les principes des droits se sont affrontés aux logiques racistes et sexistes pour que les événements jouent finalement contre les demandes des esclaves, puis contre les demandes féminines. Les $X I X^{*}$ et $X X^{c}$ siècles ne favorisent pas l'extension des droits de l'homme, combattus par les pays opposés à la France, notamment parce que mêlés aux visées expansionnistes françaises, avant d'être liés à la France des francs-maçons ou à la révolution bourgeoise, imparfaite avant celle de 1917. Il faut, là encore un événement, la seconde Gucrre mondiale et ses dévastations pour que les droits soient saisis par la communauté internationale.

En mêlant intimement les dimensions proprement culturelles des ćmotions et des sensibilités, des réactions face à la violence et la naissance de l'individualisme, aux ruptures événementielles, au hasard des luttes, Lynn Hunt donne à comprendre comment ce qui était commun aux pays des deux bords de l'Atlantique 
n'a donné suite qu'en France, où la rupture sociale a permis la reconnaissance en tant que telle de l'idéologie des droits de l'homme. Mais en retour, l'état même des relations entre les groupes confessionnels, ethniques et sexués n'a autorisé leur application qu'aux minorités protestantes, et juives avec retard, aux esclaves temporairement et jamais aux femmes. La conjonction entre idéologie et luttes politiques explique le lent délai pour leur établissement après 1948. Ce livre noue ainsi de façon tout à fait convaincante l'histoire culturelle «à l'américaine ", est-on tenté de dire pour souligner que ces mots renvoient à la prise en considération de toutes les trames qui organisent une société, avec l'histoire politique événementiclle, pour déboucher ainsi sur la résolution des questions régulièrement évoquées autour de l'unité et de la discontinuité des phénomènes historiques, autour de l'articulation entre temps court et temps long, entre idéologie et pratique, mais aussi entre les versants lumineux et sombres d'un même objet historique. Lynn Hunt n'évite pas les apories des droits de l'homme, pas plus que les conséquences inséparables de leur application dans l'établissement d'un universalisme pouvant être démobilisateur et d'un accroissement de l'individualisme. Mais c'est sur leur effet performatif qu'elle conclut, soulignant que si les droits ne résolvent pas tous les problèmes qui se posent, leur proclamation oblige cependant à les affronter, donnant ainsi la valeur à ces morceaux de papiers qui ne sont décidemment pas des pensées sans efficacité.

Jean-Clément MARTIN

Henry Heller, The Bourgeois Revolution in France, 1789-1815, New York, Bergham Books, 2006, 184 p., ISBN 1-84545-169-4, $60 \$$.

Dans la courte préface introductive à son histoire de la « Révolution bourgeoise ", Henri Heller, professeur à l'Université de Manitoba au Canada, spécialiste de la France du XVI' siècle, annonce sans ambages l'objectif assigné à ce nouveau livre : "Réaffirmer le caractère bourgeois ct capitaliste de la Révolution française ", suivant en cela la vision de Marx, elle-méme influencée par les historiens libéraux de la Restauration. Ce faisant, l'auteur entend rendre visibilité et légitimité à une interprétation marxiste de la Révolution maltraitée par plus de trente années de révisionnismes tous azimuts.

Élaborée sur la base de nombreuses lectures - principalement de travaux français récents consacrés à l'histoire économique de la Révolution auxquels l'auteur emprunte à la fois les données empiriques et les analyses - la réponse d'Henri Heller à l'historiographie " révisionniste " s'articule en trois points. Tout d'abord, il s'agit d'interroger de façon critique le bilan des révisionnismes au regard des perspectives ouvertes par les travaux d'inspiration marxistc publićs ces vingt dernières années. Pour Henri Heller, la vague révisionniste qui a frappé I'historiographie de la Révolution française trouve ses origines dans la réaction antimarxiste - à la fois idéologique et académique - des années 1970. Malgré leur grande diversité, c'est bien dans leur opposition déclarée au contenu marxiste de l'interprétation classique de la Révolution française - personnifiée, d'après l'auteur, par Albert Mathiez, Georges Lefebvre, Alhert Soboul et Michel Vovelle - que les révisionnismes ont trouvé un terrain d'entente : que l'on pense à la « théorie des élites » de Denis Richet, à la « richesse non-capitaliste " de l'Américain Georges Taylor, ou 